\title{
Direct stenting disaster bailed out by intravascular lithotripsy
}

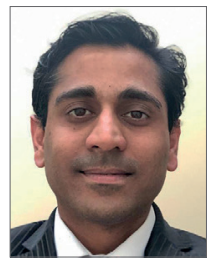

Sandeep Basavarajaiah*, MD, MRCP, FESC; Kaeng Lee, MD, MRCP;

Gurbir Bhatia, MD, MRCP; Michael Pitt,MD, MRCP

Heartlands Hospital, University Hospitals Birmingham, Birmingham, United Kingdom

This paper also includes supplementary data published online at: www.asiaintervention.org

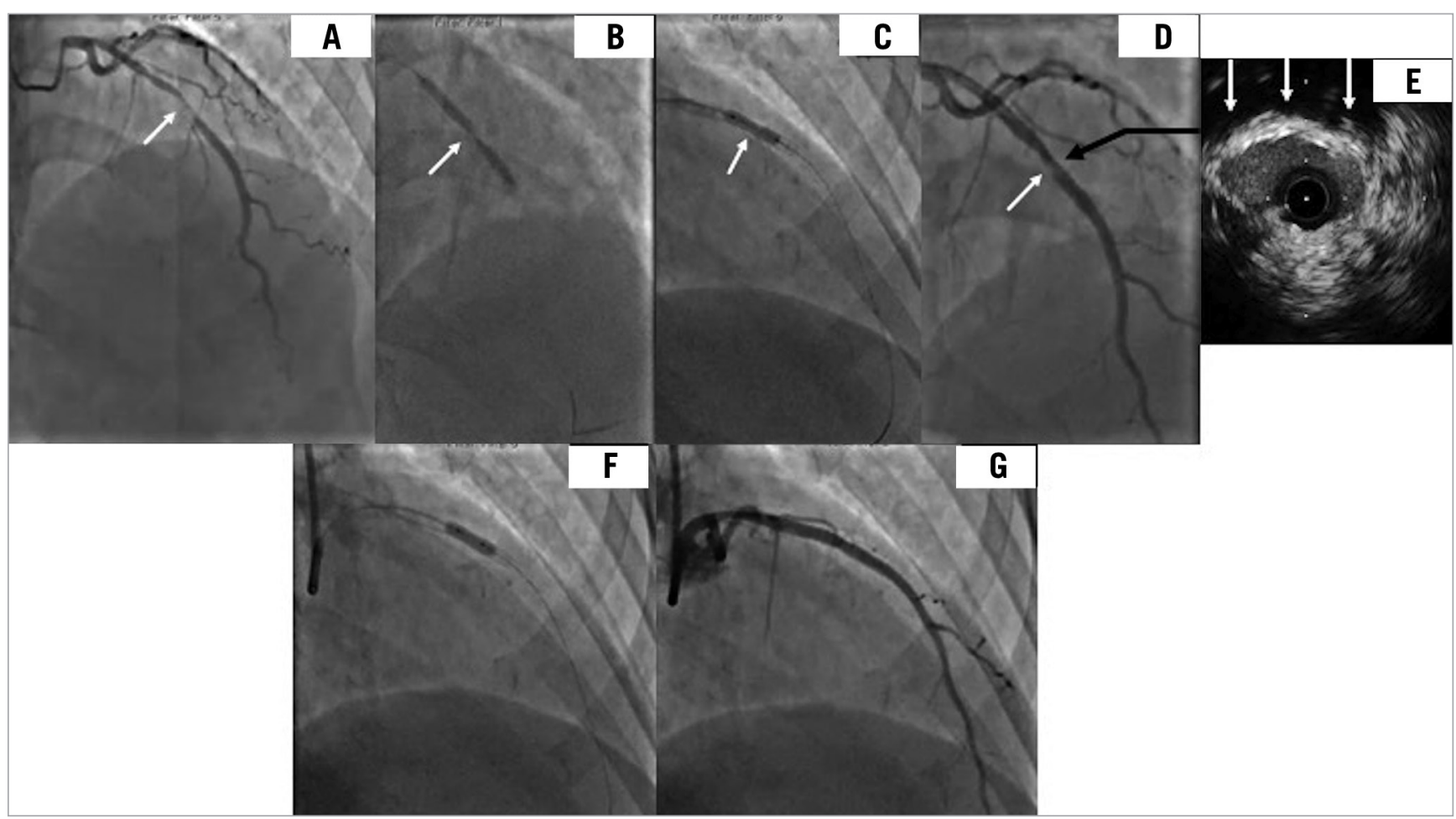

*Corresponding author: Heartlands Hospital, Birmingham, UK B9 5SS, United Kingdom. E-mail: sandeep270478@gmail.com 
A 58-year-old woman with acute coronary syndrome had a significant lesion in the mid-segment of the LAD, which appeared smooth on the angiogram (Panel A, Moving image 1). The operator directly stented the lesion $(3.0 \times 38 \mathrm{~mm}$ DES $)$ without pre-dilatation, which resulted in a focal area of significant under-expansion (Panel A \& Panel B), and which failed to expand despite using a 3.0 high-pressure non-compliant balloon inflated to $35 \mathrm{~atm}$ (Panel C \& Panel D). IVUS demonstrated the fibro-calcific nature of the lesion that resisted stent expansion and the minimum stent area was $3.5 \mathrm{~mm}^{2}$.

(Panel A-Panel E \& Moving image 2) Although, intravascular lithotripsy (IVL) is used before stent deployment in calcified undilatable lesions, there are no reports of its use in a recently deployed stent. We felt it was relatively safe to use IVL instead of largerdiameter high-pressure balloons that risked perforation. A $3.0 \mathrm{~mm}$ IVL balloon adequately expanded the stent (Panel F) and achieved a good final result (Panel G \& Moving image 3 ). The patient was discharged 24 hours later and she remains free of any clinical events at 12 months. Although there appears to be a theoretical risk of polymer and drug disruption with IVL in a recently deployed stent, in our case, we felt that it was a safer option than high pressure balloons, and we have not seen any adverse clinical outcomes at 12 months. This case reinforces the message that no lesion should be stented directly without pre-dilatation, even if it appears smooth and non-calcific on the angiogram.

\section{Conflict of interest statement}

The authors have no conflicts of interest to declare.

\section{Supplementary data}

Moving image 1. Coronary angiogram demonstrating significant disease in the mid-segment of the LAD.

Moving image 2. IVUS showing fibro-calcific lesion at the site of the under-expanded stent.

Moving image 3. Final angiogram with improved stent expansion.

The supplementary data are published online at: www.asiaintervention.org 